\title{
A Survey of Caregivers' Knowledge About Caring for Stroke Patients
}

\author{
Kyeong Woo Lee, MD, PhD, Su Jin Choi, MD, Sang Beom Kim, MD, PhD, \\ Jong Hwa Lee, MD, PhD, Sook Joung Lee, MD
}

Department of Physical Medicine \& Rehabilitation and Regional Cardiocerebrovascular Center, Dong-A University College of Medicine, Busan, Korea

Objective To investigate how much formal caregivers know about caring for stroke patients, and whether they adequately provide it.

Methods Formal caregivers, who worked for stroke patients at 8 hospitals (including 4 university hospitals, 2 rehabilitation hospitals, and 2 convalescent hospitals) participated in this study. The survey was based on a selfreport questionnaire, with 6 categories containing a total of 48 questions about the specific care of stroke patients: the demographic characteristics of the caregivers, bed positioning, the provision of meals, position changes and transfers, the range of motion exercises, and caregiver training.

Results A total of 217 caregivers were surveyed, and they were distributed as follows: $41 \%$ came from the university hospitals, $35 \%$ came from the rehabilitation hospitals, and $24 \%$ came from the convalescent hospitals. The percentages of correct answers were distributed as follows: $64.3 \%$ for bed positioning, $74.3 \%$ for providing meals, and $62.4 \%$ for position change and transfer. The total and subscale scores of the caregivers working at convalescent hospitals were significantly lower than those of the caregivers working at the other types of hospitals $(\mathrm{p}<0.05)$. Only $7.8 \%$ of the total participants received training on a regular basis. The caregivers obtained most of the information from caregiver associations $(58.1 \%)$, and the majority of the caregivers $(65.4 \%)$ were willing to receive training.

Conclusion About one third (33.8\%) of caregivers did not have adequate knowledge of how to properly care for stroke patients; in fact, a significant number of caregivers demonstrated inappropriate and insufficient knowledge in several areas. It is assumed that the provision of regular training, by rehabilitation experts, will improve the professionalism and knowledge of the caregivers, and positively affect patient outcomes.

Keywords Stroke, Caregivers, Nursing care, Surveys, Education

Received October 6, 2014; Accepted December 30, 2014

Corresponding author: Su Jin Choi

Department of Physical Medicine \& Rehabilitation and Regional Cardiocerebrovascular Center, Dong-A University College of Medicine, 26 Daesingongwon-ro, Seo-gu, Busan 49201, Korea

Tel: +82-51-240-5690, Fax: +82-51-254-8511, E-mail: mail1002zoo@hanmail.net

(c) This is an open-access article distributed under the terms of the Creative Commons Attribution Non-Commercial License (http://creativecommons.org/ licenses/by-nc/4.0) which permits unrestricted noncommercial use, distribution, and reproduction in any medium, provided the original work is properly cited. Copyright $\odot 2015$ by Korean Academy of Rehabilitation Medicine 


\section{INTRODUCTION}

A stroke is a disease with various symptoms, such as hemiplegia, sensory disorders, spasticity, balance disorders, cognitive disorders, or difficulty in swallowing. Without proper care and treatment, a stroke may result in many secondary complications, such as pressure sores, joint contracture, shoulder pains, and aspiration pneumonia. To prevent secondary complications and to support the activities of daily living (ADL) in patients, the role of the caregiver is important, particularly if the patients are in an acute stage. According to the 2011 Korean National Survey on persons with disabilities [1], 280,180 disabled people with brain lesions are currently registered, an amount that has steadily increased since 2000 . Among them, 47.9\% have severe disabilities (grades 1-2), a relatively higher proportion than other disabilities; $70.1 \%$ of the patients with brain lesions require at least moderate assistance with their ADL, while $43.6 \%$ require maximal to total assistance. Since, due to the nationwide shortage of hospital nurses, caregiver duties can include a significant portion of nursing-level tasks, the level of dependence on caregivers is higher in Korea compared to that observed in other countries [2].

This unique caregiving system in Korea, combined with the high dependence and specificity of the stroke itself results in a great burden on the caregivers of stroke patients, which means that the care of stroke patients often falls into the hands of formal caregivers rather than family members. Formal caregivers are qualified and certified professionals in the private sector; this certification includes passing a written test and the subsequent completion of 6 hours of distance education. The written test, however, does not include a detailed section on proper caregiving for stroke patients. Since the 6 hours of distance education does not include hands-on training, most formal caregivers receive additional practical training at a Caregiver Association before starting actual work. The training and practicum are usually provided by nurses, who are sufficiently qualified in terms of overall nursing care, but rarely possess specialized knowledge about strokes.

In the actual clinical setting, formal caregivers commonly provide improper care. Thus, since the care they provide may affect the outcome and secondary complications in patients, it is important to examine their knowledge base and competencies. The aim of this paper is to examine the level of knowledge possessed by formal caregivers who provide care to stroke patients (at university hospitals, rehabilitation hospitals, and convalescent hospitals) in terms of the care and services required for stroke patients.

\section{MATERIALS AND METHODS}

This survey was conducted between March and December 2013 and included 217 formal caregivers working in 8 different settings (including 4 university hospitals, 2 rehabilitation hospitals, and 2 convalescent hospitals) in the Busan area. The survey consisted of a self-report questionnaire, covering 6 categories with a total of 48 questions regarding the specific care of stroke patients (Appendix 1). The survey content included the demographic characteristics of the caregivers, bed positioning, the provision of meals, position changes and transfers, range of motion (ROM) exercises, and caregiver training. In terms of the demographic characteristics of caregivers, information regarding gender, age, and the number of years of caregiving experience, as well as the average number of patients cared for was collected. The 'Bed Positioning' category required them to select the proper position for each body part, among the supine position, side-lying position, prone position, and sitting position; a photograph and a brief description were included to aid caregiver understanding. Regarding the 'Provision of Meals' category, the questions were further divided into two categories: 'patients using a feeding tube' and 'patients requiring the use of a thickener'. For the category of 'Position Changes and Transfers', the caregivers were asked to select the correct description of a position change or transfer, and record the number of average position changes per day. ROM exercise questions examined the joints to which each ROM exercise was applied, together with the number of repetitions. The 'Caregiver Training' category surveyed whether the caregivers had received relevant training, where their source of stroke care information came from, and what the demand was like for caregiver training.

The survey focused on activities mostly performed in the hospital ward, specifically those directly associated with patients' secondary complications, or their ADL. Most of the questions, especially those pertaining to 
bed positioning, were constructed based on Krusen's Handbook of Physical Medicine and Rehabilitation (4th edition) [3], as well as DeLisa's Rehabilitation Medicine: Principles and Practice (5th edition) [4]. The questions also partially referenced Fundamentals of Current Nursing [5] and Inpatient Care for Acute Stroke Admissions [6]. SPSS ver. 12 (SPSS Inc., Chicago, IL, USA) was used for the statistical analyses. The chi-square test was used to examine the differences in the responses regarding caregiving experience and caregiver training among the hospitals. To test the differences in age, and the percentages of correct answers among the hospitals, the Kruskal-Wallis test or the Mann-Whitney U test were used. A multivariate regression analysis, in a forward stepwise manner, was used to examine the factors associated with the difference in the percentages of correct answers. Categorical variables were transformed into dummy variables. The significance level was set at $\mathrm{p}<0.05$.

\section{RESULTS}

Two hundred seventeen surveyed caregivers were distributed as follows: $41 \%$ came from university hospitals,
$35 \%$ came from rehabilitation hospitals, and $24 \%$ came from convalescent hospitals (Table 1). Joint caregivers accounted for $20.7 \%$ of the total, all of whom were working at convalescent hospitals, and three-fourths of whom were caring for at least 7 patients. All of the respondents were female, with a mean age of $58.1 \pm 5.0$ years. Those caregivers working at convalescent hospitals were, on average $53.1 \pm 5.2$ years, which is significantly younger than those working at the other hospital types $(\mathrm{p}<0.05)$. The caregivers working at convalescent hospitals had significantly less experience, compared to those working at the other hospital types $(\mathrm{p}<0.05)$.

The overall percentage of correct answers was $66.2 \%$ (64.3\% for Bed Positioning, 74.3\% for the Provision of Meals, and $62.4 \%$ for Position Changes and Transfers) (Table 2). For Bed Positioning, there was a significant difference in the percentage of correct answers, with the highest percentage $(67.8 \%)$ provided by the caregivers working at the rehabilitation hospitals, followed by those working at the university hospitals $(65.3 \%)$ and the convalescent hospitals $(57.8 \%, \mathrm{p}<0.05)$. A significant difference in the percentages of correct answers among the hospital types was also found for the category of Provi-

Table 1. The demographic characteristics of caregivers

\begin{tabular}{lccc}
\hline & $\begin{array}{c}\text { University hospitals } \\
(\mathbf{n = 8 9})\end{array}$ & $\begin{array}{c}\text { Rehabilitation hospitals } \\
(\mathbf{n = 7 5 )}\end{array}$ & $\begin{array}{c}\text { Convalescent hospitals } \\
(\mathbf{n}=\mathbf{5 3})\end{array}$ \\
\hline Age (yr) & $58.9 \pm 4.5$ & $58.5 \pm 5.0$ & $56.1 \pm 5.2$ \\
Experience (yr) & & & \\
$<3$ & 14.6 & 9.4 & 26.7 \\
$3-5$ & 21.3 & 35.8 & 20.0 \\
\hline $5-10$ & 46.1 & 52.8 & 18.7 \\
$>10$ & 18.0 & 43.4 & 5.3 \\
\hline
\end{tabular}

Values are presented as mean \pm standard deviation or percentage.

Table 2. The percentages of correct answers by category

\begin{tabular}{|c|c|c|c|c|}
\hline & All & $\begin{array}{l}\text { University } \\
\text { hospitals }\end{array}$ & $\begin{array}{c}\text { Rehabilitation } \\
\text { hospitals }\end{array}$ & $\begin{array}{c}\text { Convalescent } \\
\text { hospitals }\end{array}$ \\
\hline $\mathrm{All}^{\mathrm{a}, \mathrm{b}), \mathrm{c})}$ & 66.2 & 67.5 & 69.9 & 58.6 \\
\hline Bed positioning ${ }^{\mathrm{a}, \mathrm{b}), \mathrm{c})}$ & 64.3 & 65.3 & 67.8 & 57.8 \\
\hline Provision of meals ${ }^{a, b), c)}$ & 74.3 & 75.7 & 81.1 & 62.3 \\
\hline Position change and transfers ${ }^{\mathrm{b}), \mathrm{c}}$ & 62.4 & 65.4 & 63.1 & 56.6 \\
\hline
\end{tabular}

${ }^{a)} \mathrm{p}<0.05$ by Mann-Whitney $U$ test, between university and rehabilitation hospitals.

${ }^{b)} \mathrm{p}<0.05$ by Mann-Whitney U test, between university and convalescent hospitals.

${ }^{c)} \mathrm{p}<0.05$ by Mann-Whitney $\mathrm{U}$ test, between rehabilitation and convalescent hospitals. 
sion of Meals; $81.1 \%$ of the correct answers were given by caregivers working at rehabilitation hospitals, followed by those working at university hospitals $(75.7 \%)$ and convalescent hospitals $(62.3 \%, \mathrm{p}<0.05)$. No significant difference was observed between the rehabilitation hospitals (63.1\%) and the university hospitals (65.4\%) in terms of Position Changes and Transfers; however, the caregivers from convalescent hospitals showed a significantly lower percentage of correct answers in this category (56.6\%, $\mathrm{p}<0.05)$.

When the multivariate regression analysis was performed to examine whether other factors (excluding hospital characteristics) affected the percentage of correct answers, caregiver age was found to be one such factor $(\mathrm{p}<0.05)$. However, the statistical significance disappeared when a correlation analysis was performed between the age and the percentage of correct answers per hospital.

Most caregivers gave the following incorrect answers for the Bed Positioning category: 'place pillows under the knees in the supine position' (87.3\%), 'place the affected arm comfortably on the legs in the sitting position' (79.4\%), 'position the affected arm on the arm sling in the supine position' (76.6\%), and 'lay with the affected arm facing the top in the side-lying position, with the arm placed atop the trunk' (70.4\%) (Table 3). In most categories, the caregivers working at convalescent hospitals showed significantly lower percentage of correct answers (Table 4).

A relatively high percentage of correct answers were observed in the Provision of Meals category, although the percentage of correct answers was still significantly lower in the caregivers working at the convalescent hospitals
(Table 4). Specifically, $68.1 \%$ answered incorrectly when asked whether the patients using a feeding tube needed to remain in a sitting position for at least 30 minutes after the meal. Regarding the questions for the patients using a thickener, $62.2 \%$ of the caregivers answered that the thickener should be added to create the viscosity of a spoon-up yogurt, but only $33.2 \%$ answered that the viscosity should be that of a pumpkin porridge.

For the Position Changes and Transfers category, a high percentage of correct answers were observed overall, with almost no difference among the hospital types (Table 4). However, in regard to position change frequency, the percentage of the caregivers performing position changes every 2 hours was significantly lower in convalescent hospitals $(66.0 \%)$ than in other hospital types. Slightly less than half $(41.1 \%)$ of the caregivers answered that it is correct to lift a patient by placing one's arms underneath the patient's armpits (which is incorrect).

The ROM exercises were performed most frequently on the knee joint (70.5\%), followed by the wrist joint (67.5\%). Approximately half of the caregivers answered that they perform ROM exercises on the other joints, and that no significant difference was found among the joints. Regarding the frequency, $52.1 \%$ answered that they perform the ROM exercises 1-2 times per day, while $33.6 \%$ said that they perform ROM exercises 3-5 times per day. No significant difference was found among the hospital types.

With regard to category of Caregiver Training, 86.6\% answered that they had received at least one training session on the information in the survey, and only $7.8 \%$ answered that they receive training on a regular basis. The percentage of correct answers was significantly lower in caregivers who had received no training at all, compared

Table 3. The questions with the lowest proportions of correct answers about bed positioning

\begin{tabular}{lccc}
\hline \multicolumn{1}{c}{ Wrong bed positioning } & \multicolumn{2}{c}{ Proportion of correct answers (\%) } \\
\cline { 2 - 4 } & $\begin{array}{c}\text { University } \\
\text { hospitals }\end{array}$ & $\begin{array}{c}\text { Rehabilitation } \\
\text { hospitals }\end{array}$ & $\begin{array}{c}\text { Convalescent } \\
\text { hospitals }\end{array}$ \\
\hline $\begin{array}{l}\text { Place pillows under the knees in the supine position } \\
\begin{array}{l}\text { Place the affected arm on the legs comfortably, while in the } \\
\text { sitting position }\end{array}\end{array}$ & 13.4 & 19.4 & 4.9 \\
$\begin{array}{l}\text { Fix the affected arm with an arm sling in the supine position } \\
\begin{array}{l}\text { Place the affected arm on the trunk and lay with it atop the } \\
\text { body in the side-lying position }\end{array}\end{array}$ & 15.9 & 15.0 & 29.4 \\
$\begin{array}{l}\text { Rest feet conformably in the supine position } \\
\text { Support the wrist and forearm in an arm sling }\end{array}$ & 68.1 & 28.3 & 35.8 \\
\hline
\end{tabular}


Table 4. The percentage of correct answers about bed positioning, providing meals, and position change and transfer

\begin{tabular}{|c|c|c|c|}
\hline & \multicolumn{3}{|c|}{ Proportion of correct answers (\%) } \\
\hline & $\begin{array}{l}\text { University } \\
\text { hospitals }\end{array}$ & $\begin{array}{c}\text { Rehabilitation } \\
\text { hospitals }\end{array}$ & $\begin{array}{c}\text { Convalescent } \\
\text { hospitals }\end{array}$ \\
\hline \multicolumn{4}{|l|}{ Bed positioning } \\
\hline \multicolumn{4}{|l|}{ Supine Position } \\
\hline Ankle \& foot & 58.0 & 90.7 & 31.1 \\
\hline Hip \& knee & 71.6 & 77.8 & 53.8 \\
\hline Hand & 97.7 & 100.0 & 93.9 \\
\hline Upper limb & 64.3 & 63.0 & 62.0 \\
\hline \multicolumn{4}{|l|}{ Side-lying position } \\
\hline Hip \& knee & 59.0 & 59.9 & 61.3 \\
\hline Upper limb & 56.7 & 57.1 & 57.9 \\
\hline Prone position & 82.7 & 76.9 & 58.1 \\
\hline Sitting position & 53.1 & 57.9 & 54.2 \\
\hline Use of arm sling & 67.8 & 81.4 & 62.0 \\
\hline \multicolumn{4}{|l|}{ Provision of meals } \\
\hline \multicolumn{4}{|l|}{ Feeding tube } \\
\hline Patient's position while feeding & 87.8 & 84.1 & 82.3 \\
\hline Duration of sitting after feeding & 63.3 & 49.7 & 32.0 \\
\hline Fluid \& medication administration & 29.6 & 18.5 & 24.5 \\
\hline Oral hygiene & 59.4 & 85.9 & 38.6 \\
\hline Prevention of dry mouth & 79.2 & 85.0 & 38.6 \\
\hline \multicolumn{4}{|l|}{ Use of thickeners } \\
\hline Viscosity of drinking yogurt & 92.1 & 99.1 & 73.2 \\
\hline Viscosity of spoon up yogurt & 60.0 & 81.4 & 47.3 \\
\hline Viscosity of pumpkin porridge & 40.3 & 37.3 & 14.8 \\
\hline Fruit administration & 100 & 93.8 & 95.1 \\
\hline Medication administration & 82.5 & 86.7 & 78.0 \\
\hline \multicolumn{4}{|l|}{ Position changes and transfers } \\
\hline Use of air mattress for pressure sore prevention & 68.7 & 76.0 & 67.8 \\
\hline Effect of air mattress on sitting balance & 42.7 & 29.2 & 34.2 \\
\hline Use of air cushion for pressure sore prevention & 67.9 & 64.6 & 62.9 \\
\hline Pressure relief for patients sitting in a wheelchair & 76.9 & 85.8 & 76.5 \\
\hline Transfer technique & 52.1 & 59.8 & 56.4 \\
\hline Position change frequency & 84.3 & 88.5 & 66.0 \\
\hline
\end{tabular}

to those who had received training at least once ( $\mathrm{p}=0.05)$. The caregivers who receive training on a regular basis showed a significantly higher percentage of correct answers than those who had received training, but not on a regular basis $(\mathrm{p}<0.05)$.

When asked about their source of information, $58.1 \%$ answered that they received their information from the Caregivers Association they belonged to $24.4 \%$ said they received training from hospitals, and $15.2 \%$ acquired their information from nurses and other caregivers. For this category, the different hospital types showed different answers. Training provided by hospitals only accounted for $18.0 \%$ for university hospitals; however, a high percentage was provided by physiatrists (13.5\%). In contrast, training was provided by the hospital for $44.6 \%$ in rehabilitation hospitals. Furthermore, professional 
Table 5. The sources of caregiving information (unit, \%)

\begin{tabular}{lcccc}
\hline & All & $\begin{array}{c}\text { University } \\
\text { hospitals }\end{array}$ & $\begin{array}{c}\text { Rehabilitation } \\
\text { hospitals }\end{array}$ & $\begin{array}{c}\text { Convalescent } \\
\text { hospitals }\end{array}$ \\
\hline Training from caregiver association & 58.1 & 62.9 & 82.1 & 45.3 \\
Training provided by hospitals & 24.4 & 18.0 & 44.6 & 22.6 \\
\hline Professional materials and brochures & 8.8 & 6.7 & 16.1 & 7.5 \\
\hline Internet or non-professional materials & 0.9 & 2.2 & 0 & 0 \\
Physiatrists & 9.2 & 13.5 & 7.1 & 7.5 \\
\hline Nurses & 15.2 & 11.2 & 12.5 & 30.2 \\
\hline Caregiver colleague & 15.2 & 21.3 & 19.6 & 5.7 \\
\hline
\end{tabular}

materials and brochures also showed a high percentage (16.1\%). For convalescent hospitals, nurses accounted for $30.2 \%$ (twice the average), followed by hospital-provided training $(22.6 \%)$ (Table 5). A high demand for training was observed, since $65.4 \%$ of the respondents answered that they would be 'likely' or 'very likely' to sign up for training, if it were available.

\section{DISCUSSION}

This study examined the level of knowledge required and possessed by formal caregivers caring for stroke patients. Comparing the demographic characteristics of the three groups of caregivers revealed that caregivers working at convalescent hospitals were relatively younger, and had less work experience than those from other hospital types.

An analysis of the percentages of correct answers was conducted, according to the characteristics of each hospital type, to identify the correlation between hospital characteristics and caregiver knowledge levels. As a result, the caregivers working at convalescent hospitals showed a significantly lower percentage of correct answers across all categories; this could be explained by the fact that they were generally younger and had less experience than those working at university and rehabilitation hospitals. However, when we analyzed each hospital type separately, the percentages of correct answers did not significantly correlate with age or the number of years of caregiving experience. Furthermore, another explanation could be that the training and management of caregivers in convalescent hospitals is primarily handled by general nurses, who lack professional rehabilitation knowledge, and that the opportunities for indirect/direct training for caregivers by rehabilitation professionals are rarely available, due to the shortage of rehabilitation professionals at these institutions.

When muscles are maintained in the lengthened position, joint ROM can be maintained by reducing muscle and tissue contracture, and maintaining the sarcomere number. Therefore, proper bed positioning is an important part of stroke care, since it can help functional activities, such as the ADL or gait $[3,7,8]$.

The question in the Bed Positioning category with the highest percentage of incorrect answers was the one about 'supporting the knees with pillows in the supine position. This is a generally recommended position, since supporting the knees with pillows slightly flexes the hip and knee, thus reducing back pain. However, since it may also lead to hip and knee flexion contracture in stroke patients, this position should be avoided. Only when the hip and knees are fully extended is less muscle power required in the stance phase, since gravity is placed on the joints and ligaments [3]. Also, in the case of hip flexion contracture, hamstring muscle shortening leads to knee flexion contracture, which further results in increased ankle plantar flexion contracture, due to tightness within the gastrocnemius muscle [4].

The question with the second lowest percentage of correct answers, in the Bed Positioning category, was about 'placing the affected arm on the legs in the sitting position. This position can result in subluxation, as well as the shortening of various muscles and tissues, such as shoulder extensors, adductors and internal rotators, inferior and anterior capsules, and glenohumeral and coracohumeral ligaments [9]. In addition to this muscle and tissue shortening, shoulder adhesive capsulitis may develop as a result of the synergic patterns of muscle activation and spasticity, which may further worsen the pain and the ROM limitations.

The question with the third lowest percentage of correct 
answers was about 'fixing the affected arm with an arm sling in the supine position', while that with the fourth lowest percentage was 'lying with the affected arm facing the top atop the trunk in the side-lying position'. In both positions, the shoulders are maintained in the adduction state, and internal rotation is added when the arm is fixed with an arm sling. Previous studies have shown that the shoulder external rotation and abduction position helps maintain ROM of the shoulders [9]. An additive effect, for the ROM maintenance, can be expected by providing training on shoulder external rotation and abduction positioning in the supine or side-lying position.

Dysphagia occurs in $64 \%-78 \%$ of stroke patients; providing a proper diet in patients with dysphagia is important in order to prevent aspiration pneumonia, malnutrition, and dehydration [10].

In cases of severe dysphagia, feeding tubes are used for pharyngeal bypass; while a nasogastric tube is applied as a short-term solution, long-term use requires a gastrostomy tube. However, a pharyngeal bypass does not prevent aspiration; it may induce gastroesophageal reflux and cause secondary aspiration of the stomach contents [11]. Therefore, to minimize the risk of aspiration, the bed must be raised over a $45^{\circ}$ angle during mealtime, and for over 30 minutes after. This rule is also to be followed during the intake of small amounts of water or medication [5]. Since the aspiration of saliva cannot be prevented, oral hygiene is highly important.

Thickeners are used to control the viscosity of fluids for patients with dysphagia. The viscosity should be individualized according to the results of a videofluorographic swallow study (VFSS). The National Dysphagia Diet Task Force (NDDTF) classifies the viscosity of thickened fluids at a shear rate of 50 per second, and a temperature of $25^{\circ} \mathrm{C}$, as follows: thin (1-50 mPa.s), nectar-like (51-350 $\mathrm{mPa} \cdot \mathrm{s})$, honey-like (351-1,750 $\mathrm{mPa} \cdot \mathrm{s})$, and spoon-thick $(>1,751 \mathrm{mPa} \cdot \mathrm{s})$ [12]. Most patients with dysphagia require a honey-like or spoon-thick viscosity; in this study, this is likened to that of spoon-up yogurt or pumpkin porridge, for the purpose of localizing it to the Korean setting. Since the results of this study indicate that $66.8 \%$ of caregivers did not use the viscosity of pumpkin porridge, it appears to be necessary to provide accurate training, for caregivers dealing with severe dysphagia, on liquid viscosity. Precautions should be given, since caregivers and patients generally overlook the fact that liquid com- ing from fruits can also be aspirated.

Position change is a very important factor in the prevention of pressure ulcers. Most caregivers were knowledgeable in this category, and reported performing body position changes every 2 hours. However, in convalescent hospitals, one-third of the caregivers reported performing body position changes less frequently; this suggests that the physiatrists working at convalescent hospitals should provide more care to prevent the development of pressure ulcers. More than half (52.9\%) of the respondents answered that it is correct to lift a patient by placing both arms under their armpits. This suggests that there is a risk of shoulder injury during the transfer, and that both training and practice with physical therapists are required for the method of transferring a patient by hugging their trunk.

Alongside with bed positioning, ROM exercise is an important treatment for preventing contracture, and maintaining joint ROM. The results of this study show that ROM exercise was performed on all joints, including knee and wrist joints, at least 1-2 times per day; no significant difference between the hospital types was found, suggesting that most caregivers were generally performing sufficient ROM exercise.

In the last category, Caregiver Training, $86.6 \%$ of the caregivers answered that they had received at least one training session. Less than $10 \%$ answered that they received training on a regular basis, indicating that caregiver training was not provided systematically. There were significant correlations between the level of training and the percentage of correct answers, suggesting that providing regular training to caregivers could improve their knowledge of appropriate care for stroke patients.

In addition, the results also showed the need for training provided by rehabilitation experts, since this training was provided by hospitals or physiatrists only in $24.4 \%$ and $9.2 \%$ of the cases, respectively. By contrast, the training was provided by the respective Caregiver Associations in $58.1 \%$ of cases, and by their colleague caregivers in $15.2 \%$. This category showed a different distribution for each hospital type. For university hospitals, the training provided by the hospital was $18.0 \%$ lower than the average; however, the percentage of training provided by physiatrists amounted to $13.5 \%$, (twice that of the other hospital types. Therefore, it can be assumed that, while university hospitals do not possess a caregiver train- 
ing system, individual instruction and training is being done proactively. In contrast, the caregivers working at rehabilitation hospitals answered that the training they received was from the hospital (33.3\%), and professional materials and brochures (12\%). This finding suggests that training, or the provision of information, is being done proactively and in a systematic manner, since the caregivers working at rehabilitation hospitals showed the highest percentage of correct answers, and the difference in the level of knowledge was not substantial overall. In convalescent hospitals, $30.2 \%$ of the caregivers obtained their information from nurses; based on the fact that the nurses specializing in rehabilitation were rarely available at convalescent hospitals, it can be assumed that systematic training by rehabilitation experts was not provided.

Since $65.4 \%$ of the caregivers answered that they were willing to receive training, there appears to be a high demand for systematic and regular training conducted by rehabilitation experts (in addition to relevant publication materials), which would eventually increase the quality of care.

Until now, few detailed studies on the care of stroke patients provided by formal caregivers were available. In this context, the significance of this study resides in examining the level of knowledge about stroke patient care in formal caregivers in Korea. This study examined most of the important aspects of the specific care for 4 categories of stroke patients, provided by caregivers working in 3 types of hospitals: university hospitals, rehabilitation hospitals, and convalescent hospitals. This study attempted to provide a future direction for the training and management of formal caregivers.

However, this study has several limitations. First, our research was limited to 8 hospitals in the Busan area and its outcomes cannot be generalized to all hospitals in Korea. Second, although the questionnaire included pictures and used easy-to-understand terminology to enhance caregiver understanding, it cannot be safely assumed that the respondents maximally and accurately understood the intended meaning. Third, there could be a gap between knowledge and performance. Further research is needed to investigate how formal caregivers actually provide care for their stroke patients. Finally, although a substantial effort was made to include most important factors for improving stroke patient outcomes and preventing secondary complications, further studies may be necessary in order to investigate some of the aspects of stroke patient care that were not included in this study.

In conclusion, our results show that most formal caregivers generally do not have an appropriate level of knowledge about caring for stroke patients. The level of knowledge varies among the hospital types in which these caregivers work. Measures need to be taken, with regard to the caregivers working at convalescent hospitals, since their level of knowledge was significantly lower than those working at other hospital types. Concerning bed positioning, a significant number of caregivers demonstrated inappropriate or insufficient knowledge about several positions, which highlights the need for training. The most notable finding of this study is the demand for additional caregiver training: specifically, a significant number of caregivers in this study reported being interested in receiving training on professional care, but there was an apparent lack of supply. Thus, it can be concluded that the provision of systematic and regular training, conducted by rehabilitation experts (including physiatrists), will improve the professionalism of caregivers and positively affect the patient outcomes.

\section{CONFLICT OF INTEREST}

No potential conflict of interest relevant to this article was reported.

\section{REFERENCES}

1. Kim SH, Byun YC, Son CK, Lee YH, Lee MK, Lee SH, et al. 2011 National survey on persons with disabilities. Sejong: Ministry of Health \& Welfare, Korea Institute for Health and Social Affairs; 2011.

2. Ahn HS. The problems of privately employed caregivers and the need for novel nursing and caregiving system for inpatients. Healthc Policy Forum 2013;11:5862.

3. Kottke FJ, Lehmann JF. Krusen's handbook of physical medicine and rehabilitation. 4th ed. Philadelphia: Saunders; 1990. p. 520-8.

4. Halar EM, Bell KR. Physical inactivity: physiological and functional impairments and their treatment. In: Frontera WR, DeLisa JA, editors. DeLisa's physical medicine \& rehabilitation: principles and practice. 5 th 
ed. Philadelphia: Lippincott Williams \& Wilkins; 2010. p. 1249-72.

5. Kim JJ. Fundamentals of current nursing. Seoul: Jeong Dam Books; 2002. p. 522-74.

6. Alberta Provincial Stroke Strategy. Inpatient care for acute stroke admissions [Internet]. Alberta: Alberta Provincial Stroke Strategy; 2009 [cited 2015 Sep 15]. Available from: http://www.strokestrategyab.ca/ IP\%20Nov1909\%20Final.pdf.

7. Light KE, Nuzik S, Personius W, Barstrom A. Low-load prolonged stretch vs. high-load brief stretch in treating knee contractures. Phys Ther 1984;64:330-3.

8. Moseley AM. The effect of casting combined with stretching on passive ankle dorsiflexion in adults with traumatic head injuries. Phys Ther 1997;77:240-7.
9. Ada L, Goddard E, McCully J, Stavrinos T, Bampton J. Thirty minutes of positioning reduces the development of shoulder external rotation contracture after stroke: a randomized controlled trial. Arch Phys Med Rehabil 2005;86:230-4.

10. Martino R, Foley N, Bhogal S, Diamant N, Speechley M, Teasell R. Dysphagia after stroke: incidence, diagnosis, and pulmonary complications. Stroke 2005;36:2756-63.

11. Blumenstein I, Shastri YM, Stein J. Gastroenteric tube feeding: techniques, problems and solutions. World J Gastroenterol 2014;20:8505-24.

12. National Dysphagia Diet Task Force. National dysphagia diet: standardization for optimal care. Chicago: American Dietetic Association; 2002. 
Appendix 1. A survey of formal caregiver about caring for stroke patients

\section{Information about respondents}

1) Please state your sex and age. ( $M$ / F, years old)

2) How long have you been working as a caregiver?
(1) $<3$ years;
(2) 3-5 years;
(3) 5-10 years;
(4) $>11$ years

3) How many patients do you generally care for at a time?
(1) 1 patient;
(2) 2-3 patients;
(3) 4-6 patients;
(4) $>7$ patients

*Assume that the patient you are caring for has almost no strength in the left arm and leg. Please answer the following questions by placing " $\mathrm{O}$ " if correct or " $\mathrm{X}$ " if incorrect.

\section{Bed Positioning}

1) The following is a description of a patient in a bed position. Place an "O" or "X" for optical care.

(1) Lying Straight Facing Up

(1) Feet position

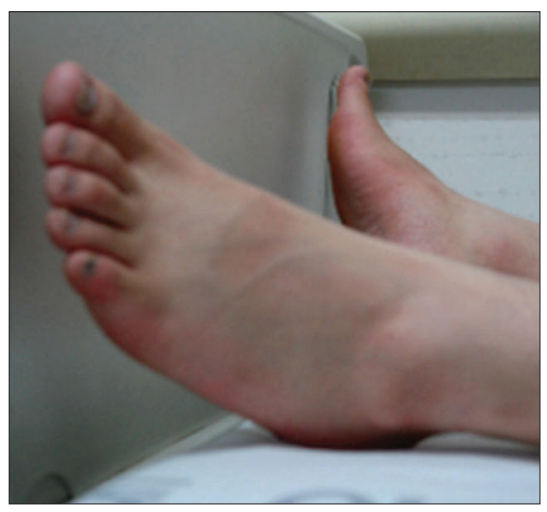

Leave feet comfortable

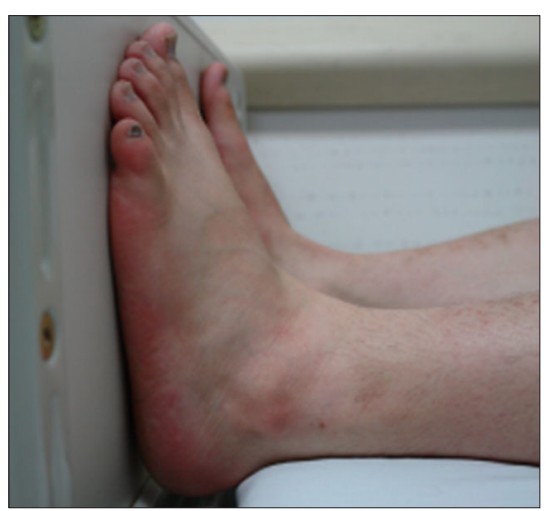

Place feet against the foot support

\section{(2) Leg position}

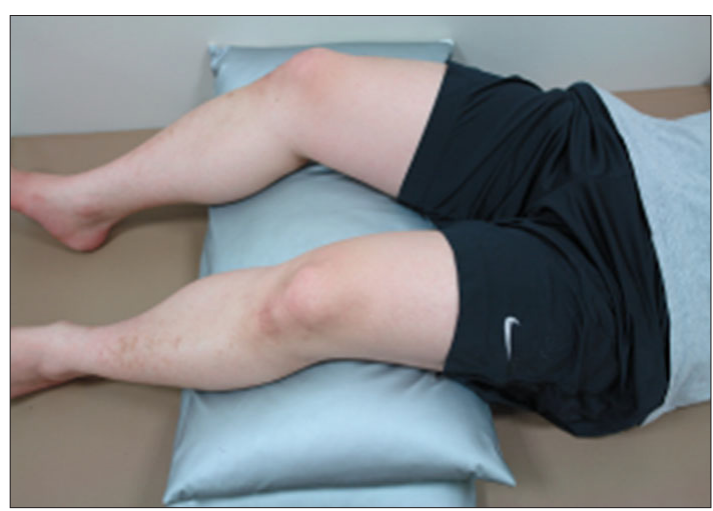

Place pillow under the knees

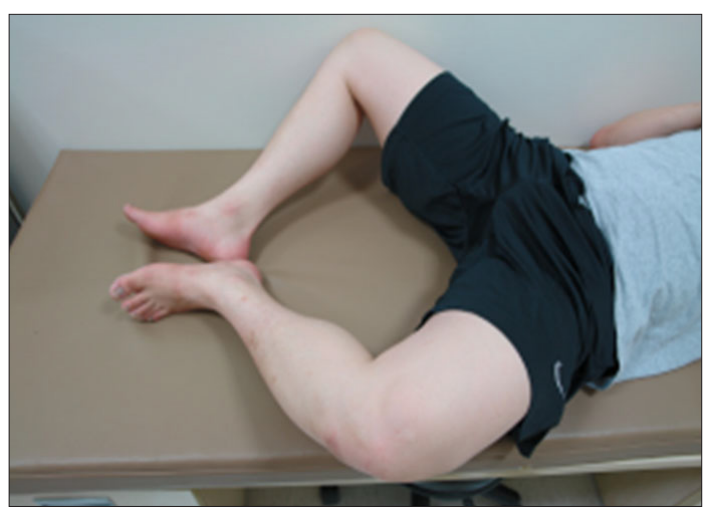

Let the leg bend comfortably 


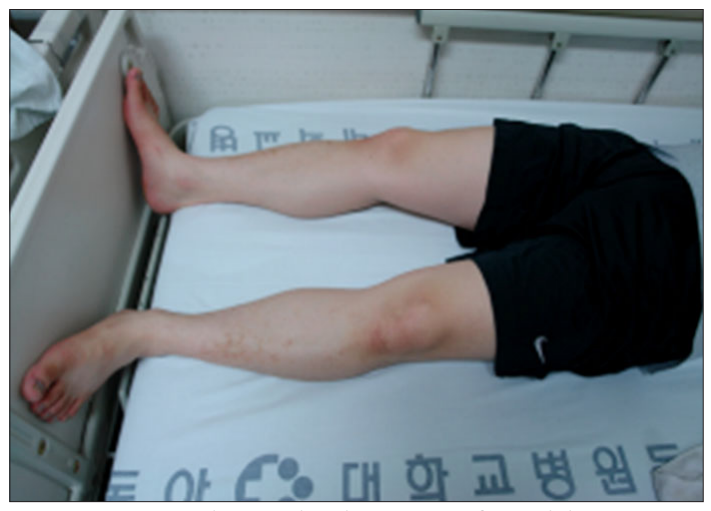

Straighten the legs comfortably

(3) Hand position

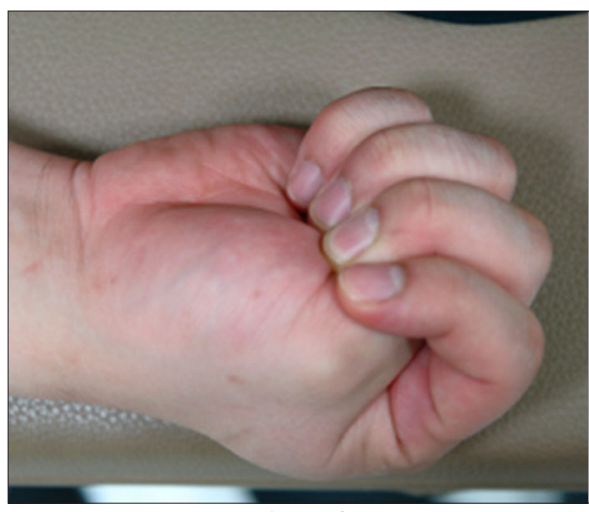

Make a fist

(4) Arm Position

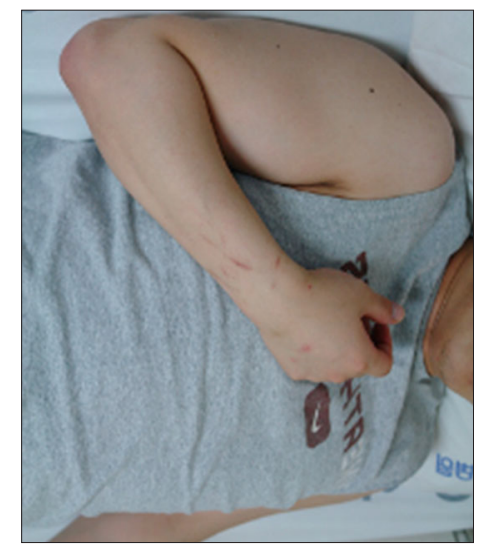

Place the limp arm atop the chest

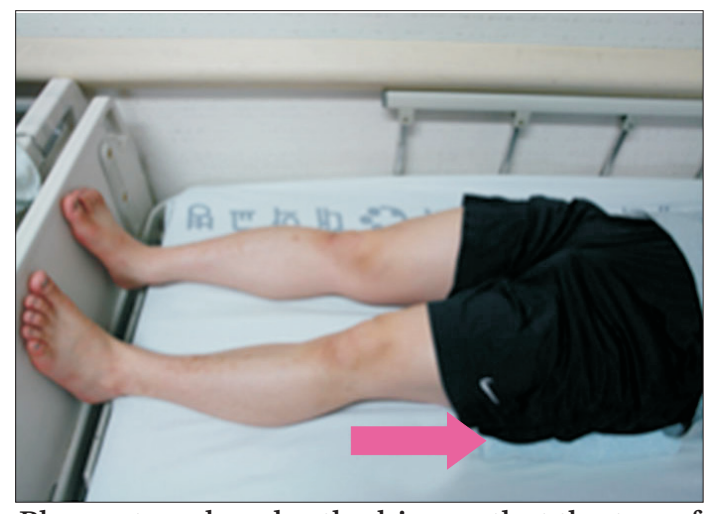

Place a towel under the hips so that the top of the foot faces upward

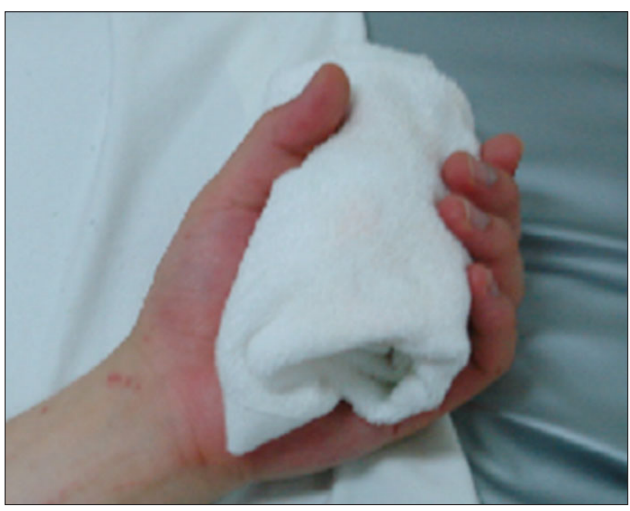

Roll hand towel and grasp it in the hand

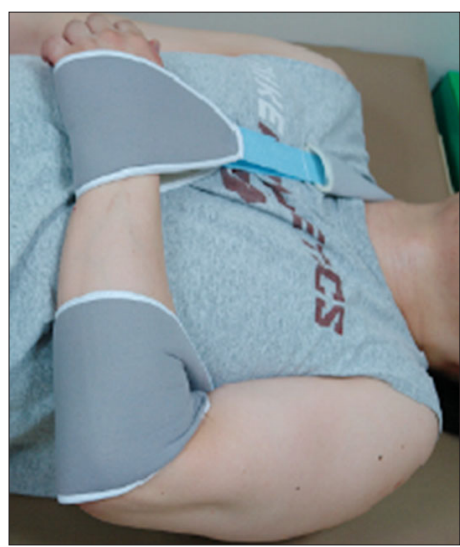

Fix the limp arm within a sling 


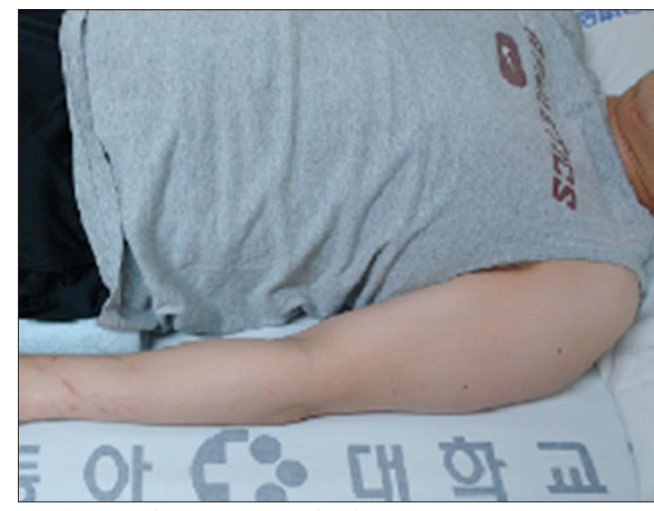

Straighten the arm and place it next to the trunk

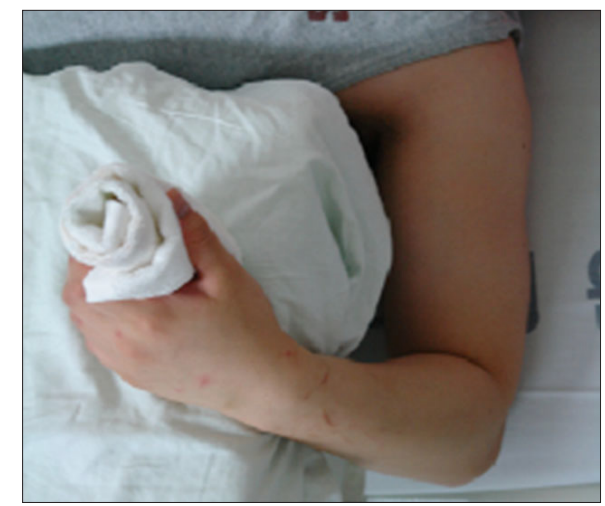

Place a pillow under the armpit and place the arm upon it

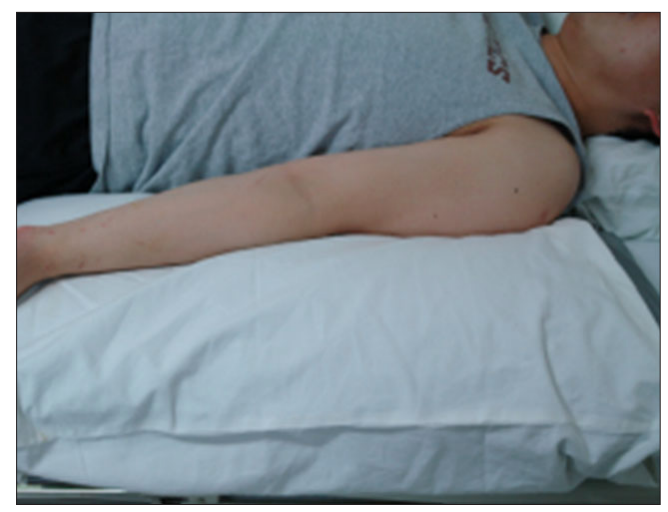

Place a pillow under the arm for support

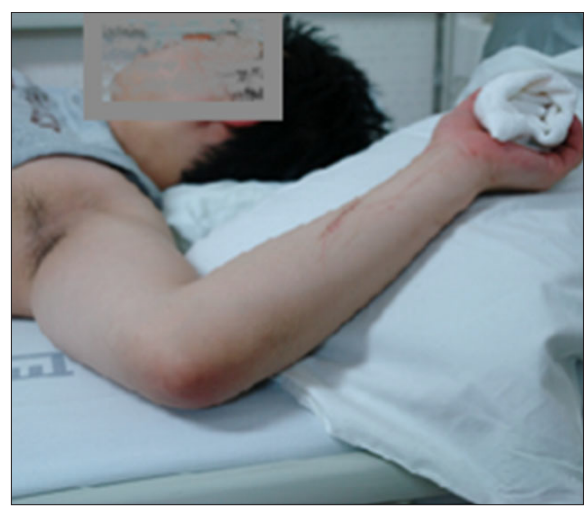

Open the arm and place the hand toward the head

(2) Side-Lying Position

(1) Leg position

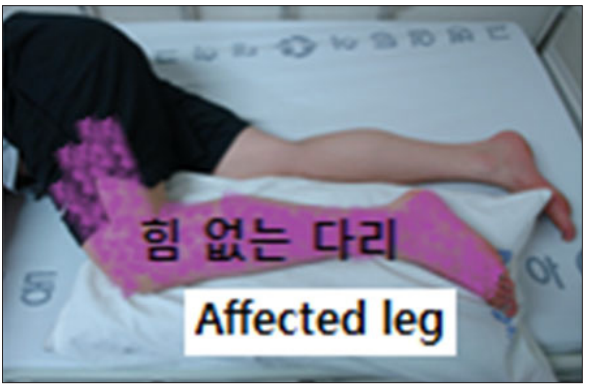

Straighten the normal leg and place the bent limp leg on the pillow

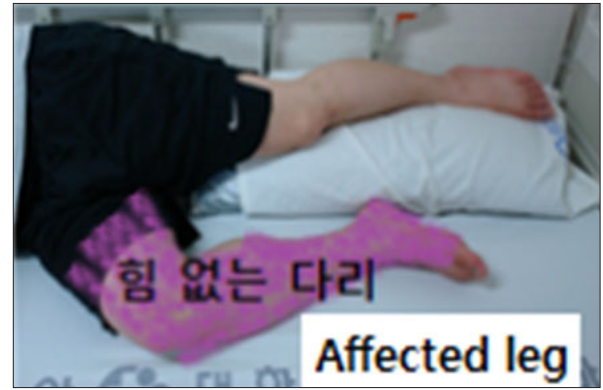

Bend the normal leg and place the straight limp leg on the pillow

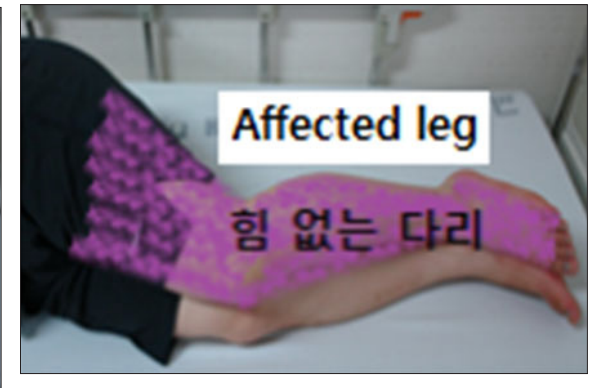

Place the limp leg atop the normal leg 
(2) Arm position

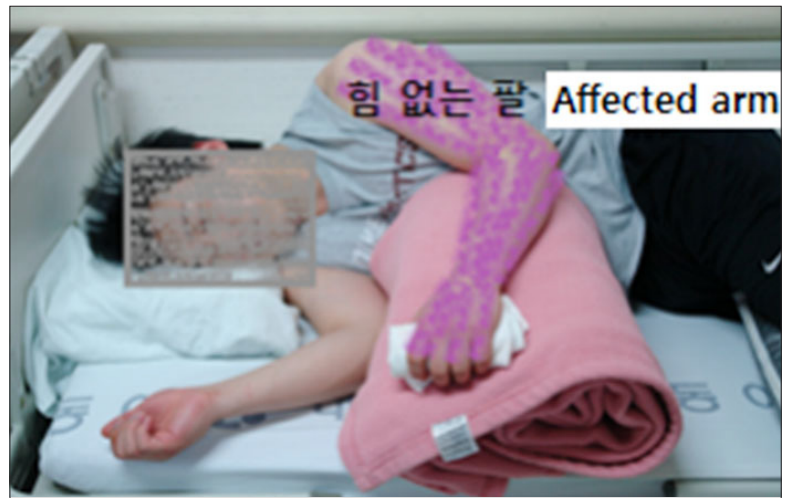

Lay with the limp arm on the top and place the pillow under the arm and twist

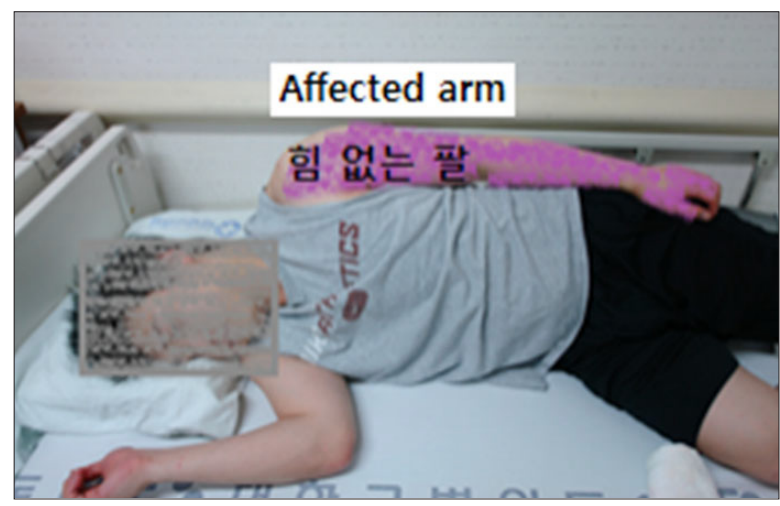

Lay with the limp arm on the top and place it atop the trunk

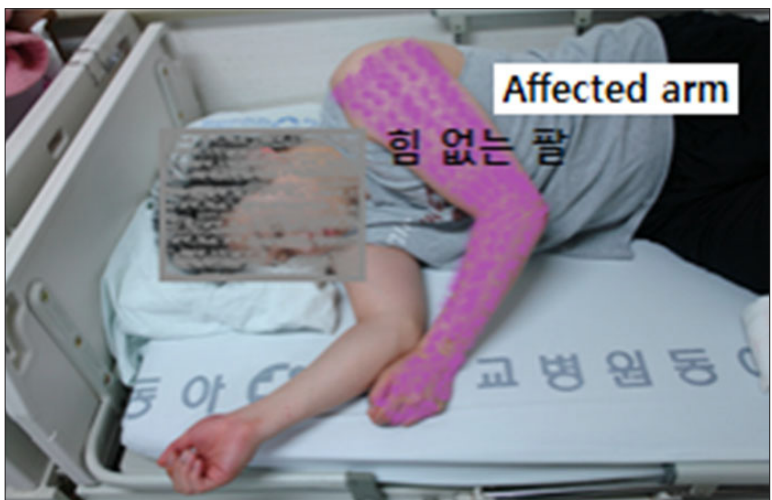

Lay with limp arm on the top and put it on the bed comfortably

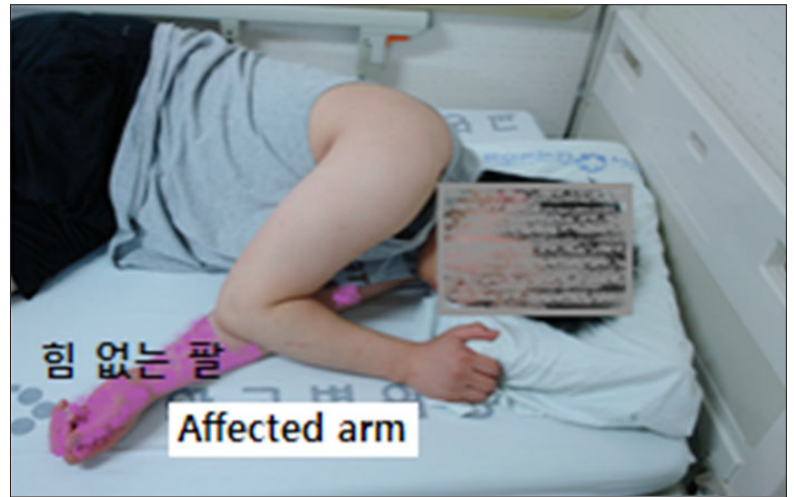

Lay with the limp arm against the bed

(3) Lying prone

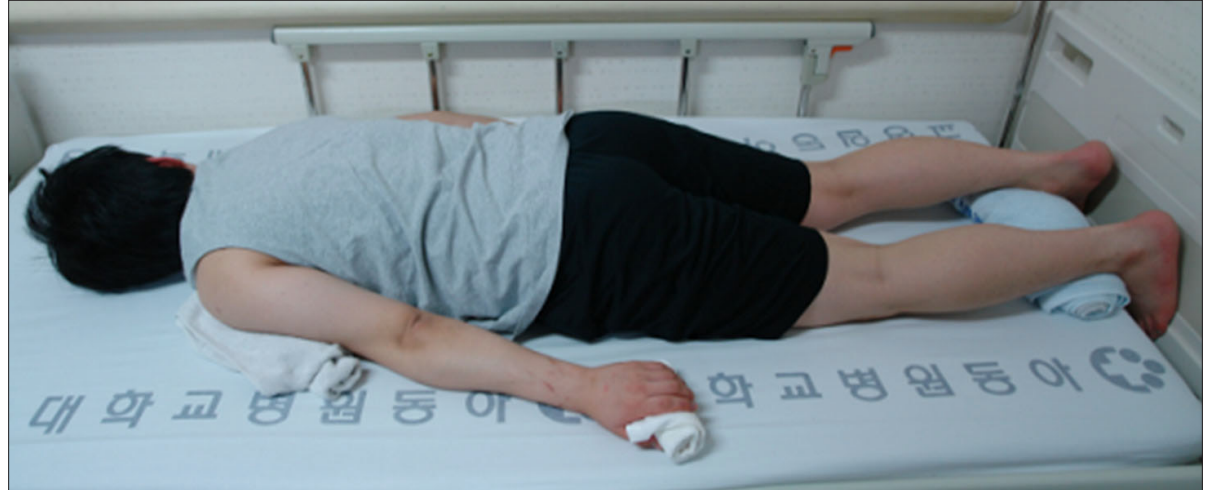

Place towels under the shoulders and ankles in the prone position 
2) The following is a description of the sitting position. Please answer the following questions by placing "O" if correct or "X" if incorrect.

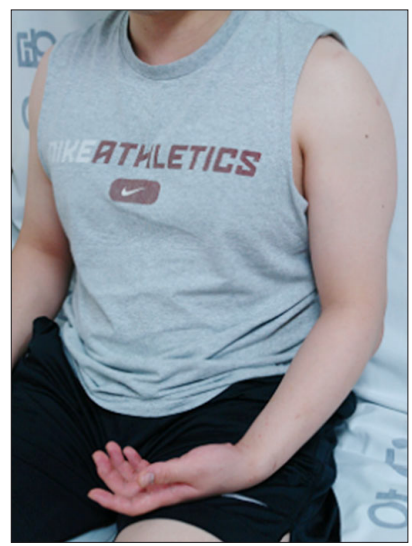

Place the limp arm comfortably on the leg

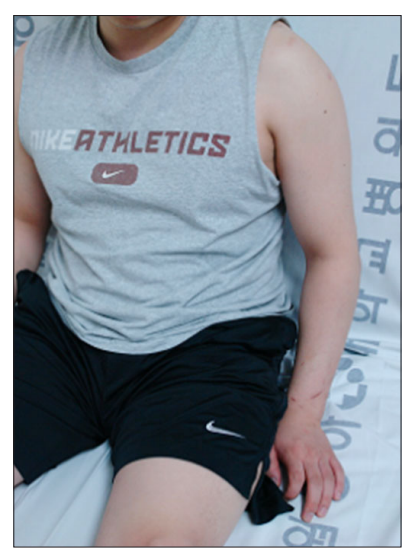

Place the limp arm comfortably on the bed

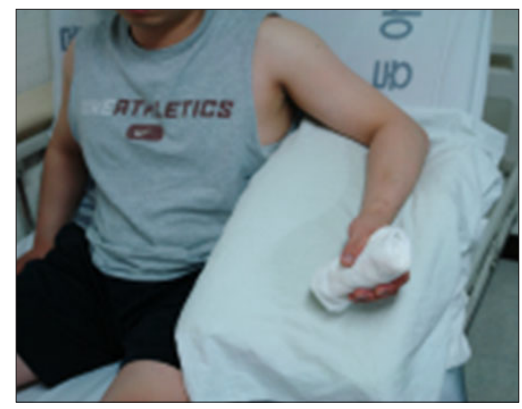

Place the limp arm atop a pillow

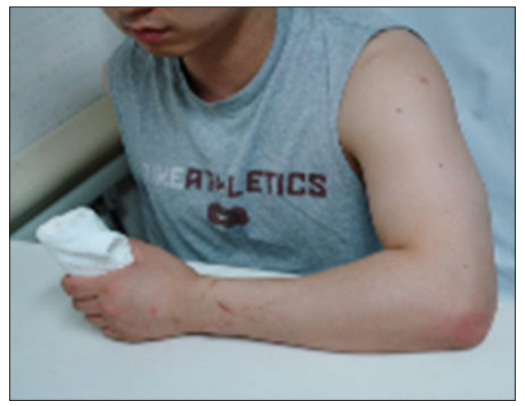

Place the limp arm on the table

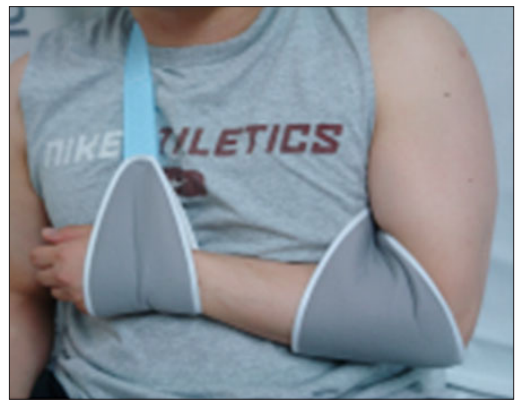

Place the limp arm within a sling

3) The following is a description of the arm in the sling. Please answer the following questions by placing "O" if correct or " $\mathrm{X}$ " if incorrect.

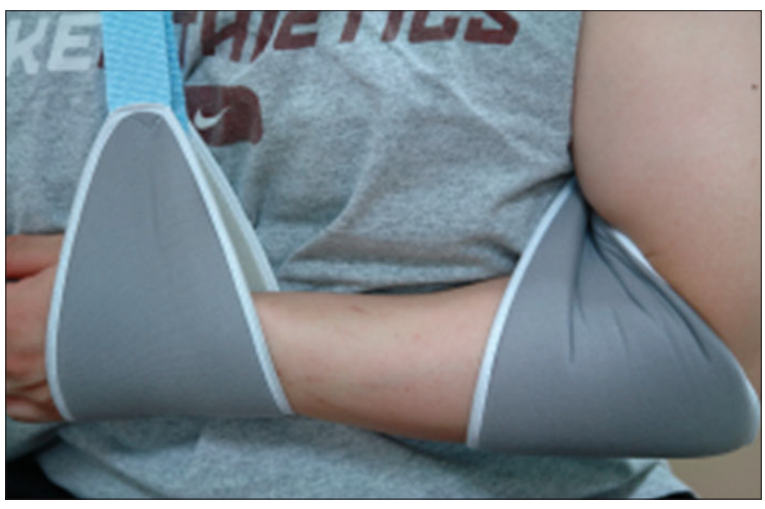

Support the wrist and the elbow

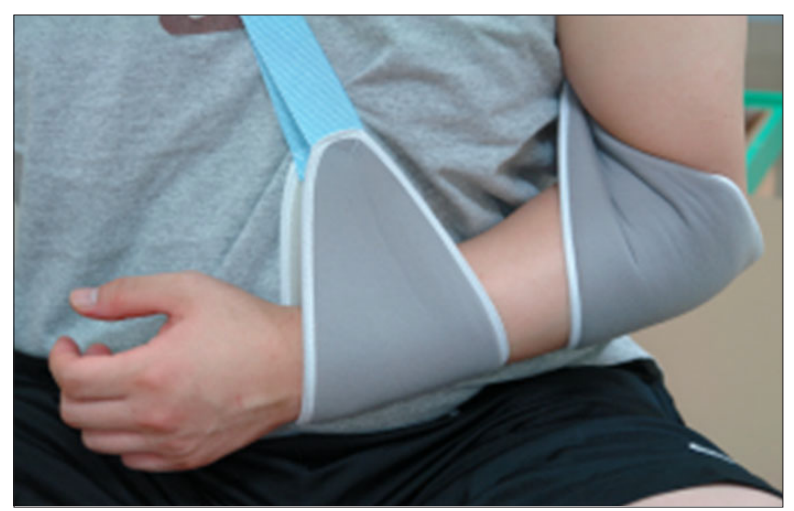

Support the forearm and the elbow 


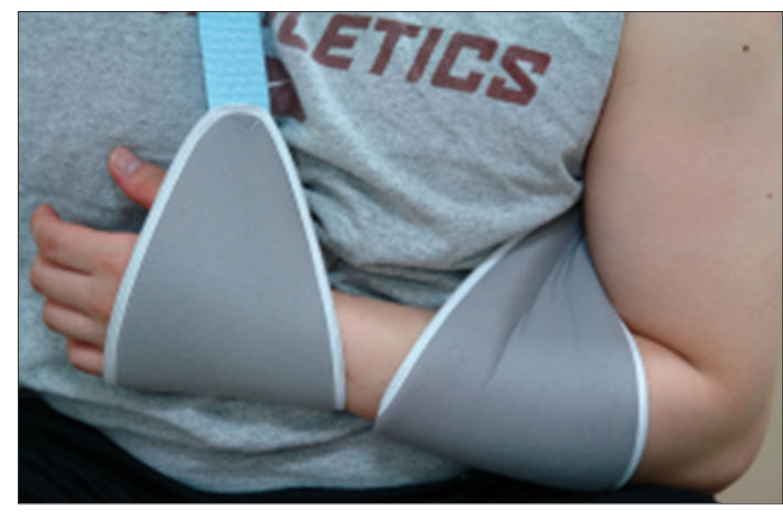

Support the wrist and the forearm

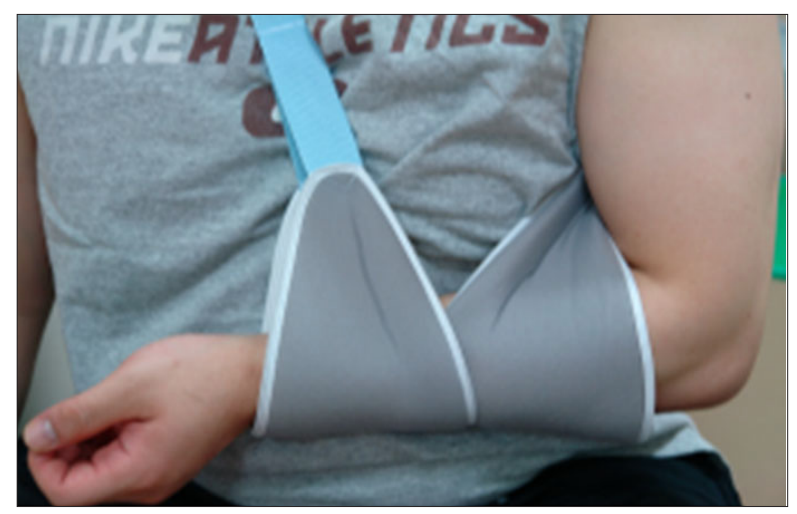

Support the forearm

\section{Providing Meals}

1) The following is a description of a patient with a feeding tube. Please select the correct answer.

(1) Height of the bed during meal
(1) lying position
(2) $<45^{\circ}$
(3) $>45^{\circ}$

(2) Time for the patient to remain in a sitting position after a meal
(1) $<5$ minutes
(2) 5-10 minutes
(3) 10-30 minutes
(4) $>30$ minutes

(3) The position to use after administering drinking water or small amounts of medicine

(1) supine

(2) elevate the bed

(3) remain in a sitting position after taking water/medicine

(4) Oral hygiene (wash inside of mouth with gauze dipped in mouthwash)
(1) no need
(2) 1-2 times per day
(3) $>3$ times a day

(5) When the inside of the mouth is dry?

(1) place wet gauze

(2) insert a small amount of water

2) The following is a description of a patient using a thickener. Please select all of the correct answers.

(1) Add thickener to create the viscosity of drinking yogurt

(2) Add thickener to create the viscosity of spoon-up yogurt

(3) Add thickener to create the viscosity of pumpkin porridge

(4) Give fruits such as apple, tangerines, or strawberries as snacks

(5) Add a small amount of water when administering medicine

\section{Position Change and Transfer}

1) Please select all of the correct answers:

(1) Pressure ulcers never occur in patients on an air mattress

(2) It is good to use an air mattress even if the patient can sit alone against the wall to prevent pressure ulcers

(3) For air cushions, inflating fully with air is better for preventing pressure ulcers

(4) There is no need to change body position while sitting in a wheelchair

(5) Lift up the patient by placing your arms under the patient's armpits when transferring them 
2) How often do you change the body position of patient when the patient is in bed?
(1) Within 2 hours
(2) Every 32 hours
(3) Rarely

\section{Range of Motion Exercises}

1) Please select all of the joints for which you apply joint exercise.
(1) Shoulder
(2) Elbow
(3) Wrist
(4) Fingers
(5) Hip
(6) Knee
(7) Ankle

2) How many times per day do you perform range of motion exercises?
(1) 0 times
(2) 1-2 times
(3) 3-5 times
(4) $>5$ times

\section{Caregiver Training}

1) Have you ever been trained on the above information?
(1) No
(2) Yes
(3) I am regularly trained

2) Where do you obtain the information regarding above?

(1) Caregiving association to which I belong

(2) Hospital

(3) Professional information or books

(4) Internet or non-professional source

(5) Doctors

(6) Nurses

(7) Other caregivers

(8) Other (Please specify):

3) Are you willing to receive training if it is available?
(1) Not at all
(2) No
(3) Maybe
(4) Very likely
(5) Yes

4) Please list below if there is any information you would like to receive about caring for patients with stroke: 